\title{
Evaluation of database-derived pathway development for enabling biomarker discovery for hepatotoxicity
}

Citation for published version (APA):

Hebels, D. G. A., Jetten, M. J. A., Aerts, H. J. W., Herwig, R., Theunissen, D. H. J., Gaj, S., van Delft, J. H., \& Kleinjans, J. C. S. (2014). Evaluation of database-derived pathway development for enabling biomarker discovery for hepatotoxicity. Biomarkers in Medicine, 8(2), 185-200.

https://doi.org/10.2217/bmm.13.154

Document status and date:

Published: 01/02/2014

DOI:

10.2217/bmm.13.154

Document Version:

Publisher's PDF, also known as Version of record

Document license:

Taverne

Please check the document version of this publication:

- A submitted manuscript is the version of the article upon submission and before peer-review. There can be important differences between the submitted version and the official published version of record.

People interested in the research are advised to contact the author for the final version of the publication, or visit the DOI to the publisher's website.

- The final author version and the galley proof are versions of the publication after peer review.

- The final published version features the final layout of the paper including the volume, issue and page numbers.

Link to publication

\footnotetext{
General rights rights.

- You may freely distribute the URL identifying the publication in the public portal. please follow below link for the End User Agreement:

www.umlib.nl/taverne-license

Take down policy

If you believe that this document breaches copyright please contact us at:

repository@maastrichtuniversity.nl

providing details and we will investigate your claim.
}

Copyright and moral rights for the publications made accessible in the public portal are retained by the authors and/or other copyright owners and it is a condition of accessing publications that users recognise and abide by the legal requirements associated with these

- Users may download and print one copy of any publication from the public portal for the purpose of private study or research.

- You may not further distribute the material or use it for any profit-making activity or commercial gain

If the publication is distributed under the terms of Article $25 \mathrm{fa}$ of the Dutch Copyright Act, indicated by the "Taverne" license above, 


\section{Evaluation of database-derived pathway development for enabling biomarker discovery for} hepatotoxicity

Current testing models for predicting drug-induced liver injury are inadequate, as they basically underreport human health risks. We present here an approach towards developing pathways based on hepatotoxicity-associated gene groups derived from two types of publicly accessible hepatotoxicity databases, in order to develop drug-induced liver injury biomarker profiles. One human liver 'omics-based and four text-mining-based databases were explored for hepatotoxicity-associated gene lists. Overrepresentation analysis of these gene lists with a hepatotoxicant-exposed primary human hepatocytes data set showed that human liver 'omics gene lists performed better than text-mining gene lists and the results of the latter differed strongly between databases. However, both types of databases contained gene lists demonstrating biomarker potential. Visualizing those in pathway format may aid in interpreting the biomolecular background. We conclude that exploiting existing and openly accessible databases in a dedicated manner seems promising in providing venues for translational research in toxicology and biomarker development.

\section{KEYWORDS: 'omics databases biomarker DILI diXa drug-induced liver injury hepatotoxicity over-representation analysis pathway development text-mining databases toxicogenomics}

\section{Hepatotoxicity assessment}

Drugs represent an important risk factor for initiating liver injury or hepatotoxicity. More than 1000 drugs and toxins have been implicated in drug-induced liver injury (DILI), which accounts for up to $10 \%$ of all adverse drug reactions $[1,2]$. Included in this is a steadily increasing number of herbal and natural supplements, which is a concerning development since these products are readily available without prescription, yet most have never been subjected to scientific testing and do not undergo any standardization of the active components [3]. The clinical presentation of DILI varies considerably and may mimic other phenotypes of acute or chronic liver disease. Acute presentations can range from mild asymptomatic liver function test abnormalities to an acute illness with jaundice that resembles viral hepatitis or acute liver failure (ALF) [4-9,101]. In fact, it is the most common cause of ALF in the USA, accounting for $20-40 \%$ of all cases $[1,10,102]$. For the majority of DILI-associated ALF cases the underlying cause is clear since it involves accidental or intentional paracetamol (acetaminophen) overdosing resulting in an accumulation of the highly reactive paracetamol metabolite $N$-acetylp-benzoquinone imine (NAPQI), which causes extensive cellular and mitochondrial membrane damage in hepatocytes followed by increased oxidative stress, eventually resulting in acute hepatic necrosis $[11-13,101]$. However, up to $16 \%$ of DILI-associated ALF cases are caused by idiosyncratic mechanisms and as such pose a significant health problem because of their poorly understood pathogenesis and potential to cause fatal outcomes (i.e., $75 \%$ of the idiosyncratic drug reactions results in liver transplantation or death) [8,14-18]. Although susceptibility to DILI is thought to be influenced by certain patient characteristics, such as age, sex, genetic predisposition, the number and type of medications, and underlying comorbidities, it remains highly unpredictable, which contributes to the fact that, currently, no specific biomarkers of idiosyncratic DILI are available [19]. There is also growing evidence that obesity predisposes to DILI [20]. Given the pandemic in obesity, DILI cases can be expected to rise in obese subjects. The rare incidence in humans (1 in 10,000-100,000) and the diverse mechanisms of toxicity complicate detection of DILI in preclinical or clinical testing and contribute to the difficulty in predicting idiosyncratic events [16].

Due to its low incidence hepatotoxicity testing in animals is a financial burden, impractical and interferes with animal welfare given the number of animals that would be needed to detect hepatotoxicity. This difficulty in detecting DILI in a preclinical setting is why in humans DILI is

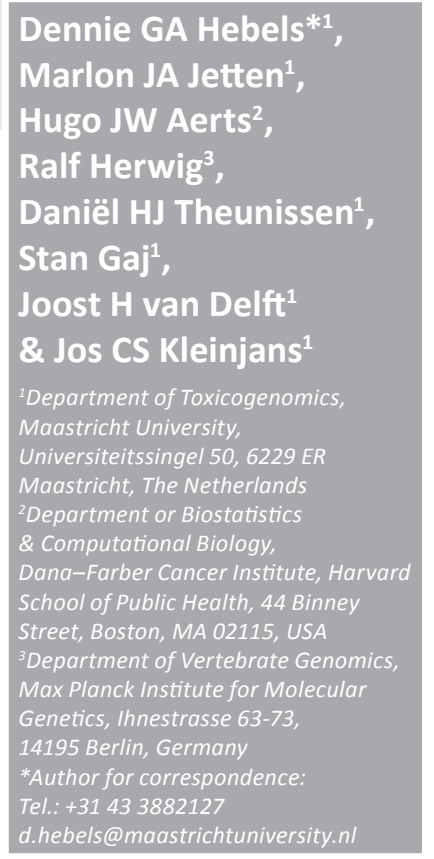

Future $\mathrm{fSS}$ 
often not detected until after the introduction of a drug to the market, making it also the most common reason for drug-related regulatory actions, including nonapprovals, restriction of use and drug withdrawal [21]. The general inadequacy of animal models to detect hepatotoxicity due to its high rate of false-negative results and the high rate in which new drugs intended for potential clinical use are currently being synthesized calls for a different strategy [15,22-33]. Elucidating the mechanisms of hepatotoxicity and predicting its occurrence has already been the aim of many studies and a very large body of information is available on this subject, especially within the area of toxicogenomics. Here, new approaches have been explored that may overcome the limitations of the current testing methods and better predict whether newly developed compounds will cause (idiosyncratic) hepatotoxicity [34-36].

Besides research papers, online publicly accessible databases also contain an enormous amount of data related to the study of liver toxicity. In particular, text-mining and 'omics databases frequently contain (published and unpublished) studies that focus on understanding the molecular mechanisms underlying hepatotoxic responses in humans. An innovative endeavor was started through the Data Infrastructure for Chemical Safety (diXa) project, funded by EU Seventh Framework Programme, to provide a single resource for the capture of data produced by toxicogenomics studies and databases, and to ensure sustainability of such a resource for use by the wider research community [103]. Such efforts are necessary, and timely, as demands for chemical safety are ongoing yet expectations are shifting away from traditional in vivo testing strategies towards increasingly computational methods, not only with regard to hepatotoxicity assessment but also in other fields of toxicology, pharmacology and molecular biology.

diXa aims at building a web-based, openly accessible and sustainable e-infrastructure for capturing toxicogenomics data, and at linking this infrastructure to available databases holding chemicological, physicological and toxicological information, and to databases on molecular medicine. As a result, this infrastructure enables the identification of possible biomarkers for safety risks. Work has so far focused on gathering this information for idiosyncratic drug-induced hepatotoxicity, but will also be extended to other pathological conditions. Users will eventually be able to analyze data available within the diXa infrastructure, as well as comparing their own data with biomarker profiles stored within the database. By exploring databases that have gathered biomolecular information on hepatotoxic exposures, new pathways may be created that are relevant for hepatotoxicity and that may be used as specific indicators of hepatotoxic mechanisms. Such profiles may be used to run dedicated 'omics analyses that will save a considerable amount of time, resources and test animals during testing of new chemicals and drugs intended for human use.

It is the purpose of this review to investigate current publicly available databases in some depth and provide an overview of what is available and how this information might be applied to obtain biomarkers for idiosyncratic hepatotoxicity based on gene lists derived from these databases. As a use case, here we present an approach towards developing hepatotoxicity-specific pathways that may be indicative of the hepatotoxicity of new chemically engineered drugs before they reach the market. Although an exploration of the possible clinical use of such biomarkers in a diagnostic context is certainly relevant, this is outside the scope of this particular review.

\section{Hepatotoxicity databases}

When attempting to find biomarkers for hepatotoxicity, the existence of publicly accessible databases that collect experiment data offers an excellent opportunity to extract possibly relevant information. Currently, data on genes or proteins that are potentially valuable as a biomarker for hepatotoxicity are offered in two types of databases:

- Databases that use text-mining approaches to identify genes or proteins that are associated with hepatotoxic conditions;

- 'Omics databases that collect 'omics data, such as transcriptomic (mRNA, miRNA and high-throughput sequencing), proteomic and metabolomic data, in some occasions with corresponding metadata.

Both types of databases could be of great value for biomarker discovery; however, they operate according to different principles. Text-mining databases use text-mining algorithms and/or manual curation to search literature repositories such as PubMed for genes and proteins that are associated with specific pathological conditions. As a result, gene lists associated with hepatotoxic conditions that are derived from text-mining tools represent a more heterogeneous approach towards biomarker discovery 
since in most cases such databases will cover and combine both in vitro and in vivo data, a wider range of biomolecular techniques (e.g., 'omics, real-time PCR, western blotting and animal knockouts), and possibly multiple species (e.g., human, mouse and rat). While 'omics databases also contain a heterogeneous collection of data, querying these databases allows for a more dedicated approach where one can target only one species and technique, for instance by selecting only human liver tissue gene expression data. Such data are particularly interesting since they most accurately represent the human in vivo pathological situation and may therefore have the best potential for developing hepatotoxicity biomarkers.

In this paper we analyzed text-mining and 'omics-based databases to review how gene lists derived from text-mining databases compare with human liver tissue-derived gene lists and how they might be used with regards to hepatotoxicity biomarker discovery in nonanimal-based cellular systems. In order to do so, we screened a selection of both types of databases for gene sets related to a number of possible hepatotoxic conditions. In Box 1 the list of search terms that was used to find suitable gene sets is displayed. Although strictly not a hepatotoxic condition, hepatocellular carcinoma was also used as a search term given the predisposition hepatocellular carcinoma has in relation to chronic liver disease [37]. Genes associated with this condition may still be related to expression changes occurring during the hepatotoxicity stages. For the same reason, hepatitis $\mathrm{C}$, cholangiocarcinoma, liver neoplasms and hepatotoxicity related to alcohol abuse were included as search terms [38-40]. After collecting gene sets, they were tested for their possible relevance in biomarker discovery in cellular liver models by performing a custom-made pathway analysis using a large transcriptomic data set on hepatotoxic compound exposure in primary human hepatocytes (PHHs), and comparing the results.

\section{- Text-mining databases}

A large number of text-mining databases can be found online. Some of them specifically focus on hepatotoxicity, while others include a wide range of pathologies. For this review, we selected four text-mining databases that include gene sets that are linked with hepatotoxicity:

- The Comparative Toxicogenomics Database (CTD) integrates data from scientific literature searched by professional biocurators, using text-mining processes to rank and prioritize articles for curation, to describe chemical interactions with genes and proteins, and associations between diseases and chemicals, and diseases and genes/proteins [104]. CTD includes curated data describing cross-species chemical-gene/protein interactions and chemical-disease and gene-disease associations to illuminate molecular mechanisms underlying variable susceptibility and environmentally influenced diseases [41,42];

- The GATACA Gene Explorer database draws information from a large number of literature, gene and disease databases (e.g., PubMed, Kyoto Encyclopedia of Genes and Genomes [KEGG], Gene Ontology [GO] and BioCarta) and functions using concept unique identifiers that understand the intended meaning of each synonymous name in each source and link all names into a single entity [105]. It enables the exploration and prediction of pathways responsible for disease causation;

- The Library of Medical Associations (LoMA) is a freely accessible database containing molecular associations of several hepatotoxic conditions [106]. This database has been established by searching the complete PubMed database by means of Medical Subject Headings $(\mathrm{MeSH})$ terms and text-mining algorithms. After filtering PubMed, the remaining publications were individually and manually validated for molecular associations [43];

- MalaCards is an integrated database of human maladies and their annotations, modeled on the architecture of the GeneCards database of human genes [107]. MalaCards provides lists of affiliated genes found to be associated textually with the key disease, using the GeneCards search mechanism and also employs manual curation of data sets [44].

After searching these databases for the search terms displayed in Box 1, associated genes per hepatotoxic condition were downloaded. Most databases provided both Human Genome Organization (HUGO) Gene Nomenclature Committee gene symbols and Entrez Gene IDs, except for MalaCards, where only HUGO Gene Nomenclature Committee gene symbols were available. Annotation conversion was carried out using the GeneCards ${ }^{\circledR}$ GeneALaCart batch-querying application [45,108].

Within the CTD, pathology-associated genes are scored according to their relevance using an 


Box 1. Hepatotoxicity search terms.
- Autoimmune hepatitis
- Cholangiocarcinoma
- Cholangitis
- Drug-induced cholestasis
- Drug-induced hepatitis
- Drug-induced liver injury
- Fatty liver disease
- Hepatocellular carcinoma
- Hepatomegaly
- Lipidoses
- Liver cirrhosis
- Liver failure
- Liver fibrosis
- Liver neoplasms
- Necrosis
- Primary biliary cirrhosis

'inference score' and whether there is any direct evidence for a molecular marker/mechanism association between the disease and the gene. Only those genes with this direct evidence were extracted from the database. In LoMA, GATACA and MalaCards the complete lists of associated genes were used. In Table 1 , an overview is presented of all gene lists (CTD: 11; GATACA: ten; LoMA: seven; and MalaCards: ten) that were extracted from the four text-mining databases.

\section{- 'Omics databases}

Public 'omics databases are mainly represented by ArrayExpress [109] and the Gene Expression Omnibus (GEO) [110], developed and maintained respectively by the European Bioinformatics Institute and the National Center for Biotechnology Information [46,47]. Both databases contain functional genomics experiments that can be queried for specific pathological or experimental conditions, species, etcetera, and data can be downloaded for personal use. They include gene expression data from microarray and high-throughput sequencing studies while data have been collected according to the Minimum Information About a Microarray Experiment (MIAME) and Minimal Information about a high-throughput SEQuencing Experiment (MINSEQE) standards. GEO experiments are also imported within the ArrayExpress database.

ArrayExpress was searched for the hepatotoxicity terms described in Box 1 and only studies analyzing human liver tissue specimens were selected. In the majority of selected studies, the associated publication was found through
ArrayExpress or GEO, and gene lists reported by the authors to be associated with the hepatotoxic condition under investigation were extracted. Where needed annotation conversion was performed using GeneALaCart. A total of 14 studies were included, covering 18 hepatotoxicityrelated gene lists [48-61]. In TabLe 2, an overview is given of all gene lists that were extracted from the selected studies and the hepatotoxic condition they are associated with. All lists of genes were derived from the tables and the supplementary data provided in the publications. Data analysis in these publications was performed using significant analysis of microarray analysis, predictor of microarray analysis, linear modeling or t-test analysis, and in all cases the lists of significantly modified genes passed a Benjamini-Hochberg false-discovery rate cutoff of 0.01 or 0.05 .

\section{Over-representation analysis of database gene lists}

The next step in evaluating the relevance of hepatotoxicity databases for biomarker prediction in in vitro models was the assessment of their relevance by performing an over-representation analysis on the extracted gene lists. In order to do so gene lists were first converted to a GenMAPP Pathway Markup Language (GPML) format within Cytoscape, an open source software platform for visualizing complex gene networks [62,63]. GPML is a custom XML format compatible with gene list/pathway visualization and analysis tools such as Cytoscape, GenMAPP and PathVisio [64,65], the latter of which was used to perform a gene list over-representation analysis (based on z-scores) with a publicly available test set of transcriptomic data related to hepatotoxicity. The over-representation analysis employed by PathVisio assesses whether the number of differentially expressed genes (DEGs) in the hepatotoxicity test set in a given gene list (r) compared with the total number of genes in that gene list (n) is significantly higher than the background ratio of the total number of DEGs in the hepatotoxicity test set (R) compared with the total number of measured genes in the hepatotoxicity test set $(\mathrm{N})$, that is, it determines if there is an over-representation of the number of DEGs belonging to a particular gene list, compared with what could be expected by chance alone. This is calculated as a z-score, which represents a measure of relative deviation of $r$ from its expected mean value: $r-(n * R / N)$ divided by the standard deviation. Its associated $\mathrm{p}$-value calculation is based on the hypergeometric distribution. In the analysis presented here using 
a hepatotoxicity test set, a gene list with a high z-score would thus indicate that it contains more DEGs than would be expected by chance. Such a gene list would therefore be of interest for its biomarker potential since it is over-represented by the DEGs in the hepatotoxicity test set.

\section{Hepatotoxicity test data set}

For over-representation analysis of results obtained from in vitro liver models, gene expression data from $\mathrm{PHH}$ s exposed to 64 different hepatotoxic compounds were selected from Toxicogenomics Project-Genomics Assisted Toxicity Evaluation system [111], a large-scale public database of transcriptomic and pathology data potentially useful for predicting the toxicity of new chemical entities [66]. The selection of these 64 compounds was based on overlap with the list of compounds for which human liver toxicity data (in the form of a DILI severity score or potential) are made available by the US FDA in their Liver Toxicity Knowledge Base. A list of these 64 compounds is displayed in Box 2. For all compounds the full gene expression data set, which included a maximum of three exposure times (2, 8 and $24 \mathrm{~h}$ ) and three doses (low, middle and high), and their corresponding vehicle controls, was downloaded from the database and a MAS5 condensing algorithm was used to normalize the data. Nonexpressed probes were removed from the data set and further filtering was performed by selecting only genes that were significant for at least one of the 64 compounds in a linear model analysis that included exposure time and dose as fixed variables (unadjusted $\mathrm{p}<0.01$ ). We subsequently continued with data from the $24 \mathrm{~h}$ middle- and high-dose exposures since most gene expression changes were found to take place under these conditions. From this filtered data set, DEGs were defined as having an absolute $\log ^{2}$ ratio of 0.5 (i.e., the ratio of the gene expression level of compound-exposed $\mathrm{PHH}$ s versus the vehicle control gene expression level, followed by a $\log ^{2}$ transformation to create a symmetric distribution).

In PathVisio, the DEGs were subsequently used to perform an over-representation analysis per compound and calculate the corresponding z-scores as described earlier. Database-derived gene lists were considered to be significantly overrepresented at $\mathrm{p}<0.01$ (equivalent to $\mathrm{z}>2.58$ ). The results of the over-representation analysis are shown in Supplementary Tables $1 \& 2$ (see online at www.futuremedicine.com/doi/suppl/10.2217/ bmm.13.154) for the text-mining gene lists and the human liver 'omics gene lists respectively. In these tables, all calculated z-scores per compound and dose for each gene list are presented and significant $\mathrm{z}$-scores (i.e., $\mathrm{z}>2.58$ ) are colored in red. The $z$-scores of the text-mining and human liver 'omics gene lists were subsequently used to perform a clustering analysis, which builds a hierarchy based on z-score pattern similarity. The $\mathrm{z}$-score table is reordered in such a way that similarly scoring gene lists and compounds are clustered together. The hierarchical cluster shown in Supplementary Figure 1 visualizes the z-score results. The average numbers of significant gene list hits per database are summarized in Supplementary Table 3 and visualized in Figure 1. A high average level of significant hits indicates a strong overrepresentation in the $\mathrm{PHH}$ hepatotoxicity test data set of gene lists in that particular database. In Figure 1A this is shown as the average number of significant gene lists per dose group and in Figure 1B as the average number of significant compounds per gene list. The corresponding calculations are shown in Supplementary Tables $1 \& 2$.

\section{Database comparison: text mining versus human liver 'omics}

The general pattern observed in the results from the over-representation analysis is that

\section{Table 1. Text-mining database gene lists.}

\begin{tabular}{|lllll} 
Hepatotoxic condition & $\begin{array}{l}\text { CTD } \\
(\mathbf{n})\end{array}$ & $\begin{array}{l}\text { GATACA } \\
(\mathbf{n})\end{array}$ & $\begin{array}{l}\text { LoMA } \\
(\mathbf{n})\end{array}$ & $\begin{array}{l}\text { MalaCards } \\
\text { (n) }\end{array}$ \\
\hline Autoimmune hepatitis & - & - & 27 & - \\
\hline Cholangiocarcinoma & 6 & 98 & 275 & 696 \\
\hline Cholangitis & 3 & 12 & 61 & - \\
\hline Drug-induced liver injury (cytotoxicity) & 41 & - & - & - \\
\hline Drug-induced cholestasis & 43 & 45 & - & 209 \\
\hline Drug-induced hepatitis & - & - & - & 22 \\
\hline Hepatitis & 21 & 169 & - & 3055 \\
\hline Hepatocellular carcinoma & - & - & 539 & 2031 \\
\hline Hepatomegaly & $6566^{+}$ & 123 & - & - \\
\hline Lipidoses & 41 & 43 & - & 7 \\
\hline Liver cirrhosis & 138 & 142 & - & 335 \\
\hline Liver failure & - & 50 & - & - \\
\hline Liver fibrosis & - & 70 & 157 & 195 \\
\hline Liver neoplasms & 154 & - & - & - \\
\hline Necrosis (not liver specific) & 10 & - & - & - \\
\hline Primary biliary cirrhosis (cholestasis) & - & - & 60 & 259 \\
\hline Steatosis (fatty liver disease) & 10 & 9 & 90 & 200 \\
\hline $\begin{array}{l}\text { The CTD hepatomegaly gene list is the only gene list where an inference score cutoff }>20 \text { was used } \\
\text { to create a larger gene list. } \\
\text { CTD: Comparative Toxicogenomics Database; LoMA: Library of Medical Associations. }\end{array}$ & & & & \\
\hline
\end{tabular}


Table 2. List of recent 'omics data publications found in ArrayExpress from which 18 hepatotoxicity-associated human liver gene lists were extracted.

\begin{tabular}{|c|c|c|c|c|c|}
\hline Study (year) & PMID & ArrayExpress ID & Hepatotoxic condition(s) & Genes (n) & Ref. \\
\hline Wurmbach et al. (2007) & 17393520 & E-GEOD-6764 & Cirrhosis & 8 & [61] \\
\hline Onomoto et al. (2011) & 21603632 & E-GEOD-11190 & Nonresponding hepatitis $C$ & 31 & [56] \\
\hline Caillot et al. (2009) & 19477948 & E-GEOD-11536 & Fibrosis progression & 16 & [51] \\
\hline $\begin{array}{l}\text { Bourd-Boittin et al. } \\
\text { (2011) }\end{array}$ & 21826695 & E-GEOD-24667 & Fibrosis associated with hepatitis C or alcohol abuse & 68 & [50] \\
\hline Liu et al. (2011) & 21931690 & E-GEOD-24807 & Nonalcoholic steatohepatitis & 1552 & [53] \\
\hline Andersen et al. (2012) & 22178589 & E-GEOD-26566 & Cholangiocarcinoma & 238 & {[} \\
\hline Affò et al. (2013) & 22637703 & E-GEOD-28619 & Alcoholic hepatitis & 102 & {$[48]$} \\
\hline \multirow[t]{2}{*}{ Sia et al. (2013) } & 23295441 & E-GEOD-32225 & $\begin{array}{l}\text { Intrahepatic cholangiocarcinoma associated with } \\
\text { inflammation }\end{array}$ & 160 & [58] \\
\hline & & & $\begin{array}{l}\text { Intrahepatic cholangiocarcinoma associated with } \\
\text { proliferation }\end{array}$ & 1402 & \\
\hline \multirow[t]{2}{*}{ Starmann et al. (2012) } & 23071592 & E-GEOD-33814 & Steatohepatitis & 41 & [59] \\
\hline & & & Steatosis & 23 & \\
\hline Rasmussen et al. (2012) & 22278598 & E-GEOD-34798 & Severe fibrosis & 35 & [57] \\
\hline Nissim et al. (2012) & 23185381 & E-GEOD-38941 & Acute liver failure & 643 & [55] \\
\hline \multirow[t]{2}{*}{ Staten et al. (2012) } & 23270325 & E-MEXP-2589 & Fibrosis & 7 & [60] \\
\hline & & & Fibrosis-linked inflammation & 12 & \\
\hline \multirow[t]{3}{*}{ Lake et al. (2011) } & 21737566 & E-MEXP-3291 & $\begin{array}{l}\text { Phase I and II drug-metabolizing enzymes, phase } 0 \\
\text { uptake transporters and phase III efflux transporters } \\
\text { associated with: }\end{array}$ & & [52] \\
\hline & & & Nonalcoholic steatohepatitis & 262 & \\
\hline & & & Steatosis & 26 & \\
\hline Marshall et al. (2013) & 23527199 & E-MTAB-950 & $\begin{array}{l}\text { Hepatocellular carcinoma associated with hepatitis B and } \\
\text { C or hemochromatosis }\end{array}$ & 17 & [54] \\
\hline
\end{tabular}

human liver 'omics-based gene lists outperform text-mining-based gene lists. The human liver 'omics database gene lists score a higher average number of hits per dose group than any of the individual text-mining database gene lists (Figure 1A). In addition, high doses on average score approximately twice as high as the middle doses. When considering the average number of hits across all gene lists (for all compounds and doses), only the CTD lists score similar to the human liver 'omics lists (Figure 1B). However, it should be kept in mind that this average is based on a lower number of gene lists for CTD compared with human liver 'omics (11 and 18 respectively, see Tables $1 \& 2$ ), thus rendering the human liver 'omics gene lists still higher scoring. It becomes apparent that gene lists derived from 'omics data from human liver, that is, a setting that is as close to the human in vivo state as possible, is preferable when attempting to identify gene lists that may serve as a marker of hepatotoxicity. This is also illustrated by the bottom clustering branch on the vertical axis in SUPPLEmenTary Figure 1 (indicated in green), which contains the gene lists with the highest z-scores (indicated in red in the heat map) and which are most strongly represented in a large number of high dose hepatotoxic compounds. Out of the 15 gene lists within this branch eight belong to a human liver 'omics data-based gene list (out of a total of

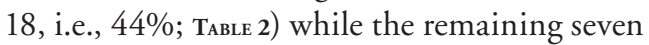
text-mining database gene lists (out of a total of 38, i.e., 18\%; TABLE 1) almost exclusively consist of CTD and MalaCards gene lists. It is therefore obvious that the human liver 'omics data-based gene lists are disproportionately highly represented in the high scoring cluster. It also shows that the performance of the text-mining gene lists, with regard to the average number of significant hits, differs strongly between the four text-mining databases (up to $\sim$ sevenfold between CTD and GATACA, Figure 1A \& 1B). Another interesting observation is that approximately half of the gene lists in this cluster are based on 
search terms that are not strictly DILI associated, indicating that also genes related to nonDILI hepatotoxicity show promise as potential biomarkers.

While the size (i.e., the number of genes) of the high-scoring gene lists in the bottom cluster ranges considerably, the 'omics data-based gene lists contain lower numbers (ranging from 17 to 643 genes) compared with the text-miningbased gene lists (ranging from 138 to 2031). This could be an indication that gene lists from 'omics data represent a more specific response, which is especially interesting in terms of biomarker development based on dedicated 'omics analyses.

In the horizontal compound axis of the hierarchical clustering analysis, two main branches are formed. The right branch contains mostly compounds with a high exposure level, which is also accompanied by a higher frequency of significant hits (indicated in red), while the middle concentrations are mostly located in the left branch. The high doses of compounds therefore seem to elicit the strongest gene expression responses and are most strongly over-represented in the high scoring gene list cluster which also corresponds with the difference in average significant hits between middle and high doses seen in Figure 1A.

\section{Critical considerations \\ Noise in text-mining approaches}

The better performance of gene lists retrieved from human liver 'omics data may be explained by the fact that these represent hepatotoxic responses in vitro more closely than the heterogeneous gene lists derived from text-mining databases, which not only contain information from dozens to possibly hundreds of different studies, but also mix in vitro and in vivo experiments and (possibly unintentionally) different species. While text-mining-based approaches seem to be quite promising for discovering new biomarkers and, as a result of the method used, cover a lot of ground, they might be prone to wrong interpretations during the curation process, assigning genes to a hepatotoxic category for which the proof is limited, thus creating 'noise' in the data. The human liver 'omics-based gene sets are all derived from single studies and the genes included within the list are all selected based on false-discovery rate cutoff criteria. However, it has to be considered that human liver tissue is also prone to inter-individual differences at the gene expression level, and as such will not always represent a uniform response when the number of liver samples is limited, which is often the case due to difficulties obtaining such tissue $[67,68]$.
When examining the clustering dendogram more closely it is apparent that gene lists for the same hepatotoxic condition but generated from different databases do not cluster together. It would thus appear that these gene lists do not contain the same genes. While the numbers of genes present within each list already suggests this (Taвle 1), this dissimilarity also points at a low overlap between gene lists. Indeed this is the case, as illustrated by the Venn diagrams in Figure 2, demonstrating that the overlap between the same hepatotoxic conditions from different databases is very poor. While the text-mining methods in the databases reviewed here differ with respect to the algorithms used and any manual curation will undoubtedly be subject to differences too, it is still a surprising observation with important ramifications. It seems wise to

Box 2. All 64 hepatic compounds selected from the

Project-Genomics Assisted Toxicity Evaluation system database

based on overlap with the Liver Toxicity Knowledge Base.

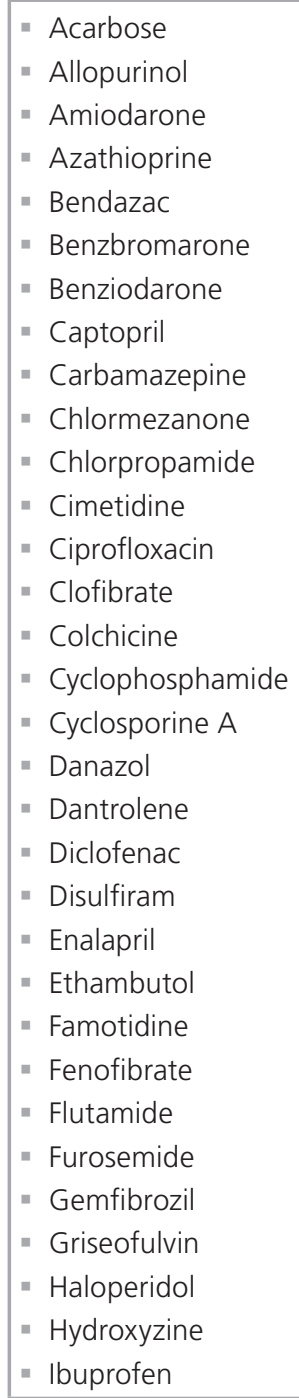

- Imipramine

- Indomethacin

- Iproniazid

- Isoniazid

- Ketoconazole

- Labetalol

- Methimazole

- Methyldopa

- Mexiletine

- Moxisylyte

- Naproxen

- Nicotinic acid

- Nifedipine

- Nimesulide

- Nitrofurantoin

- Pemoline

- Penicillamine

- Perhexiline

- Phenobarbital

- Phenytoin

- Propylthiouracil

- Ranitidine

- Rifampicin

- Simvastatin

- Sulindac

- Tacrine

- Tamoxifen

- Terbinafine

- Tetracycline

- Ticlopidine

- Tolbutamide

- Valproic acid 


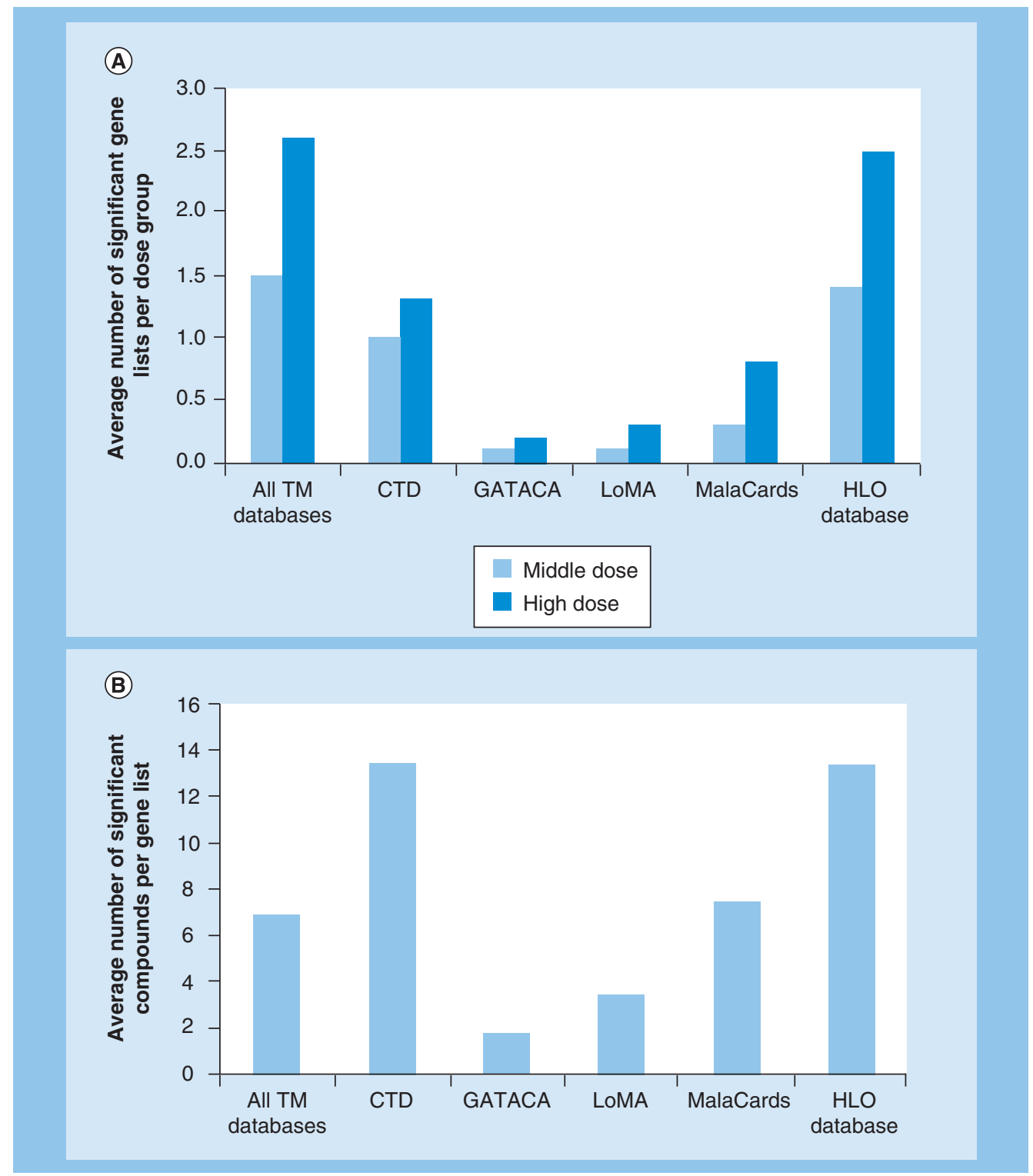

Figure 1. Average number of significant gene lists per dose group and significant compounds (middle and high dose combined) per gene list after over-representation analysis for each database or group of databases. (A) Significant gene lists per dose group; (B) significant compounds (middle and high dose combined).

CTD: Comparative Toxicogenomics Database; HLO: Human liver 'omics; LoMA: Library of Medical Associations; TM: Text mining.

be cautious when using information from textmining databases. While this does not automatically mean that this information is of less value, a better approach might be to just consider genes that overlap between different text-mining databases and therefore may represent a more robust signal. A careful examination of how text-mining databases work may also be helpful for determining where the most reliable signals can be obtained from. If we consider the four text-mining databases evaluated here, CTD and MalaCards might be preferred. Figure 1, clearly shows that gene lists from CTD and MalaCards outperform GATACA and LoMA if the numbers of significant hits are considered. It is therefore not surprising that the bottom clustering branch in SuPplementary Figure 1 , besides human liver 'omics based gene lists, mainly contains CTD and MalaCards-based gene lists. CTD and MalaCards both employ sophisticated text-mining algorithms and are both extensively curated and kept up to date with progress in the field $[41,44]$. While LoMA also employs curation, this database has not been updated since late 2009 . The GATACA database has no accompanying publication that describes their method of work 
in more detail and there is no mention of manual curation on the GATACA website. This is a strong indication that text-mining approaches towards database buildingheavily rely on continuous updating and accurate manual curating of found associations in order to be of value for biomarker discovery.

\section{- Liver injury classification}

While not shown here, pathway z-scores were also correlated (Spearman's $\rho$ ) with DILI scores developed by the FDA [69]. DILI scores represent a classification system for hepatotoxic compounds to assess their DILI potential. A high
DILI score (representing a severe hepatotoxic response) might therefore be reflected by a high z-score indicative of a strong cellular response to the hepatic injury. However, we could not find any statistically significant association $(\mathrm{p}<0.01)$ between z-score levels and DILI scores. This may be explained by the fact that DILI scores are based on hepatic injury that often only appears after long-term drug usage (usually weeks or months) by humans. The short-term exposure of only $24 \mathrm{~h}$ in the PHHs possibly does not elicit a response that reflects long-term exposure and therefore may not correspond with the DILI classification system.

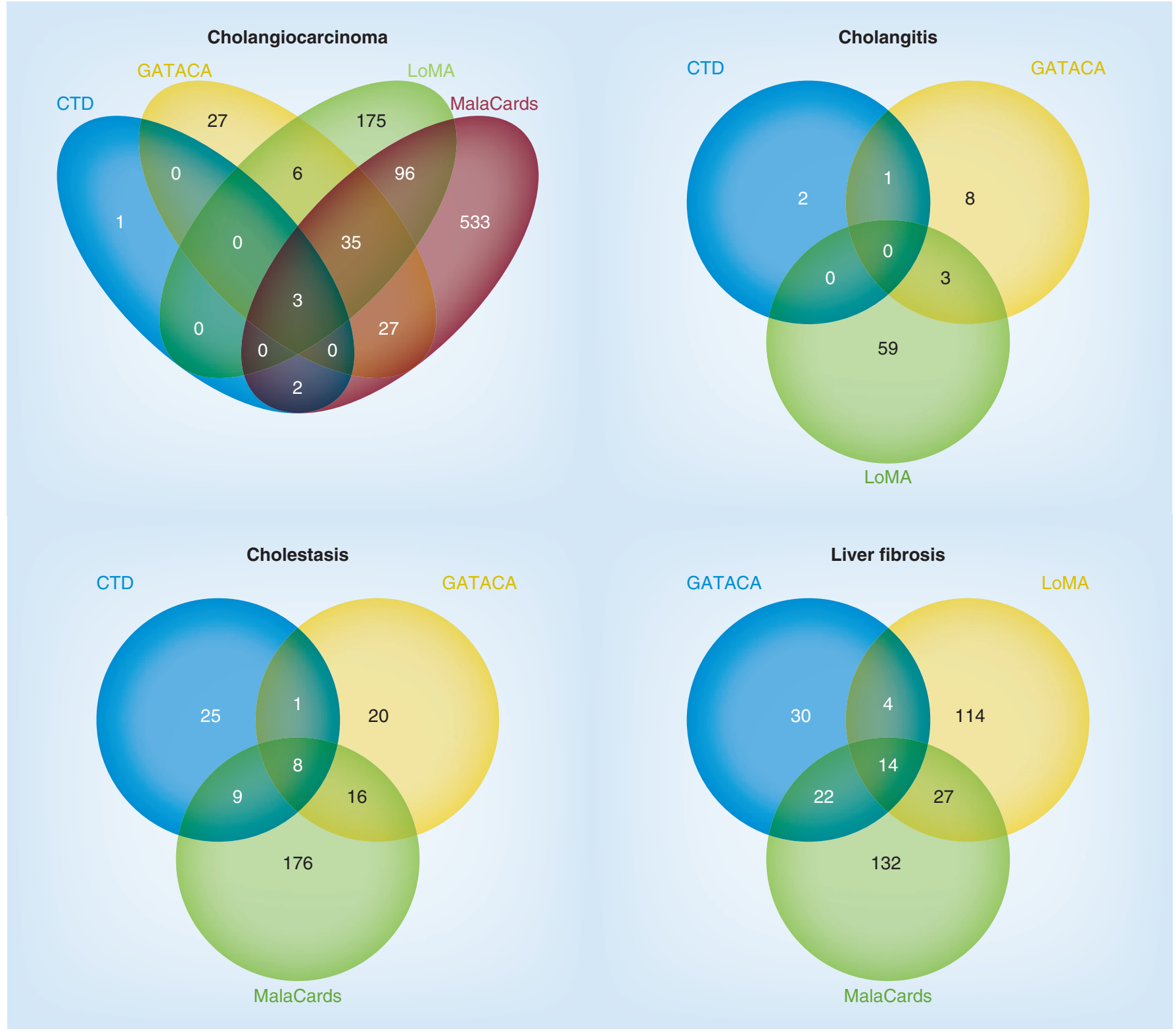

Figure 2. Four examples of the limited overlap between text-mining database gene lists representing the same hepatotoxic condition.

CTD: Comparative Toxicogenomics Database; LoMA: Library of Medical Associations. 


\section{PHHs as a test data set}

The Project-Genomics Assisted Toxicity Evaluation system hepatotoxicity test data set we used to assess the relevance of the constructed pathways as possible biomarkers was derived from PHHs. However, these cells have some drawbacks such as their limited lifespan and availability. Furthermore, within the human population large interindividual differences in response to xenobiotic exposure are known to exist, which are often related to differences in the expression or activity levels of xenobiotic metabolizing enzymes and transporters $[67,68]$. To reflect human exposure most accurately, a data set based on fresh human liver; for example, liver slices or even perfused liver, exposed to hepatotoxic compounds would need to be used. However, given the low availability of human liver this is difficult to realize and unpractical to perform experiments with [70]. $\mathrm{PHHs}$ have been used as a model system for the human liver for many years now, and while it is an in vitro system, it is the closest routinely used alternative to in vivo liver currently available [71]. $\mathrm{PHH}$ can be isolated from liver tissue, and have the advantage that they can be cryopreserved and cultured in a sandwich culture simulating the natural environment in the liver, without drastic functional changes compared with in vivo hepatocytes [72-74]. With regard to cellular processes such as biotransformation, DNA-damage response, apoptosis and cell cycle regulation, $\mathrm{PHHs}$ resemble human liver considerably better than commonly used liver cell lines such as HepG2 and HepaRG [75-79]. Therefore, PHHs are considered as a prime model for collecting molecular and mechanistic information for the evaluation of hepatotoxicity responses in humans [80]. For drug development purposes, PHHs also represent a crucial experimental model, allowing an early evaluation of human drug properties to guide the design and selection of new drug candidates while simultaneously increasing the probability of clinical success [81].

\section{Gene list visualization in pathway format}

After identifying potentially interesting gene lists (i.e., high-scoring gene lists in the overrepresentation analysis) it may be helpful to visualize these in pathway format to assist the biological interpretation. To accomplish this, network creation tools such as Cytoscape are very useful $[62,63]$. Cytoscape offers the possibility of building networks using a network building plugin. This plugin, called Michigan Molecular
Interactions, gathers data from well-known protein interaction databases and displays the interaction networks and attributes [82-84]. Interaction networks are created using several different algorithms integrated within the Michigan Molecular Interactions plugin. Depending on the number of input genes (the query genes, i.e., a gene list extracted from a database) and whether intermediate genes (i.e., neighbors) are allowed to become part of the network, the following algorithms are available to create a network:

1 Query genes + nearest neighbors;

2 Interactions among query genes (i.e., no neighbors are added);

3 Query genes + neighbors' neighbors;

4 Nearest neighbors shared by more than one query gene.

After successful creation of a network, the networks can be exported as GPML files, which can later be used for pathway analyses. The GPML format is also used to store pathway content at WikiPathways, which is an open collaborative platform dedicated to the curation of biological pathways [85]. The pathway building workflow is illustrated in Figure 3, using one of the high-scoring human liver 'omics gene lists (E-GEOD-33814, steatohepatitis) as an example.

\section{Conclusion \& future perspective}

In this review we have examined an approach to use publicly available databases that have gathered liver toxicity data, in order to develop biomarkers for hepatotoxicity derived from in vitro liver systems, whichmay potentially be used to identify hepatotoxicity of newly developed drugs. After selecting hepatotoxicity-specific gene lists from four text-mining databases and a (human liver) 'omics database, we investigated the relevance of these gene lists by performing an over-representation analysis with a large data set containing transcriptomic data of $\mathrm{PHH}$ exposed to a large number of hepatotoxic compounds. The results show that the overlap of gene lists extracted from some online text-mining-based databases is limited and that their performance in the over-representation analysis differs greatly. In particular, gene lists extracted from the GATACA and LoMA databases appear to be of little use while CTD- and MalaCards-based gene lists perform much better.

It seems likely that the performance of textmining-based gene lists is strongly influenced 


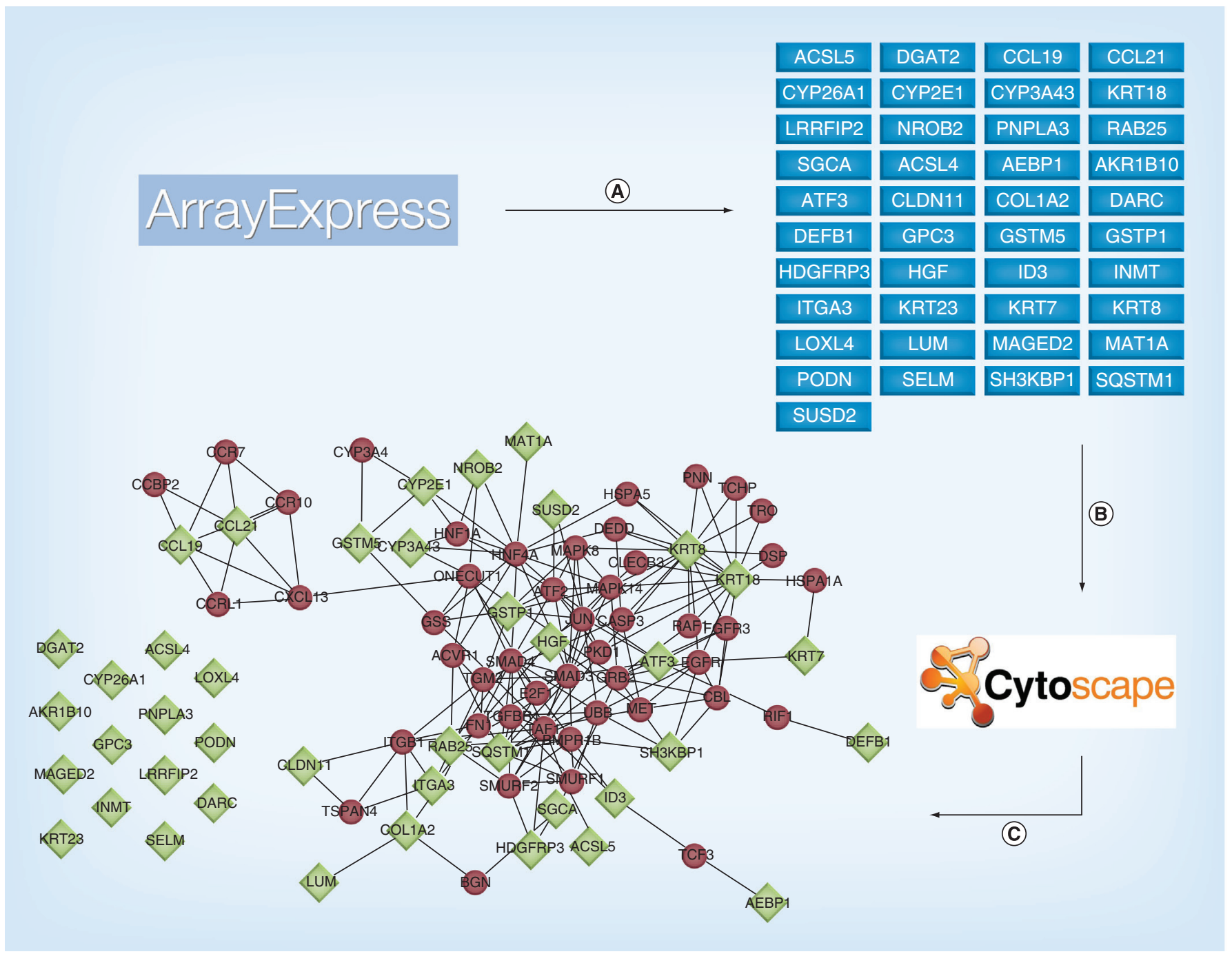

Figure 3. From gene list to pathway, illustrated for the E-GEOD-33814 steatohepatitis gene list. (A) Gene lists are first downloaded from a database (in this case ArrayExpress). (B) The gene list is imported into Cytoscape. (C) A pathway is created in Cytoscape using the Michigan Molecular Interactions networking plugin. Algorithm 4 (nearest neighbors shared by more than one query gene) was used to construct the network. In the pathway the original input genes are displayed as diamond-shaped gene nodes, while added neighbor genes are displayed by circle-shaped gene nodes.

by the efficiency of the text-mining algorithm to find the right associations, the ability to query many different data repositories, a thorough curation process performed by biomolecular experts and continuous updates. 'Omics databases have the advantage of being able to select just hepatotoxicity studies that use human liver, thereby investigating the process of human liver injury in vivo. This is also reflected by a better performance of these gene lists in the analysis presented in this paper, although the CTD and MalaCards text-mining gene lists also appear highly relevant given their average significant hit rate and should therefore certainly not be dismissed.

However, using 'omics databases to obtain human liver data also has some disadvantages. First, the availability of such data is limited to the scarcity of human liver samples. This limits the number of specific hepatotoxic conditions to choose from and puts a restriction on the sample size, and thus statistical power. Another important point is that interindividual differences create variability in the data, which complicates the finding of a robust response. This may be overcome by pooling data from all available studies and performing an overall statistical analysis.

Still, the approach used here towards discovering new biomarkers shows that both databases yield results that may be useful. While a preference might be given to human in vivo data, the vast amount of data related to hepatotoxicity in animal and in vitro studies presents a source of information that can be highly relevant. Indeed, in many studies animal and in vitro data have been shown to 
be of relevance for understanding the process of hepatotoxicity and the services provided by the diXa project will enable a comparison of all these data sources to find the most robust and reliable genes with regard to human toxicity testing [32,76,77,86-92]. Using an over-representation analysis to find gene lists that both reflect exposure to hepatotoxicants and are linked with hepatotoxicity provides a specific signature that could be used for the identification of the (idiosyncratic) hepatotoxic potential of newly developed compounds. Although the aim of this review was to provide a way of finding signatures related to the prediction of hepatotoxicity development, these same signatures may also prove to be useful in diagnosing hepatotoxic conditions in early stages of disease development. This is especially relevant since there are currently no specific biomarkers of idiosyncratic DILI and its clinical presentation can mimic many other hepatological disorders associated with, for example, alcohol abuse and

\section{Executive summary}

\section{Hepatotoxicity assessment}

- Drug treatment is an important initiator of drug-induced liver injury, which accounts for up to $10 \%$ of all adverse drug reactions. It is the most common cause of acute liver failure in the USA, accounting for $20-40 \%$ of all cases.

- Animal models are prone to misclassifying human hepatotoxicity.

- Publicly accessible databases contain an enormous amount of data related to the study of liver toxicity and offer a possibility for a different strategy to elucidate the mechanisms of hepatotoxicity and predict its occurrence.

- A change of strategy was initiated with the Data Infrastructure for Chemical Safety (diXa) project, which aims to provide a single resource for capturing data produced by toxicogenomics studies and databases, and ensure sustainability of such a resource for use by the wider research community.

- diXa aims to build a web-based, openly accessible and sustainable e-infrastructure for capturing toxicogenomics data, and to link this to available databases holding chemico-/physico-/toxico-logical information and databases on molecular medicine.

- As a result, this infrastructure enables the identification of possible biomarkers for exposure and disease.

- Here we review the applicability of pathways created based on hepatotoxicity-associated gene lists derived from hepatotoxicity databases to develop biomarker profiles and ultimately integrate them into the diXa data warehouse.

\section{Hepatotoxicity databases}

- To find lists of genes associated with hepatotoxicity, databases that use text-mining approaches (Comparative Toxicogenomics Database [CTD], GATACA, Library of Medical Associations [LoMA] and MalaCards) and 'omics databases that collect 'omics data (ArrayExpress) were explored and gene lists were collected.

- The numbers of gene lists per database were: CTD, 11; GATACA, ten; LoMA, seven; MalaCards, ten; and ArrayExpress, 18.

- The gene lists' relevance was assessed by performing an over-representation analysis on the extracted gene lists with a gene expression data set from primary human hepatocytes exposed to 64 different hepatotoxic compounds (middle and high dose, $24 \mathrm{~h}$ of exposure).

\section{Over-representation analysis of database gene lists}

- High doses of compounds elicited stronger responses than the middle doses, on average scoring approximately twice as high.

- Human liver 'omics-based gene lists performed better than text-mining-based gene lists and were strongly represented in the most highly activated cluster of a hierarchical clustering analysis, containing 15 gene lists that comprised $44 \%$ of all human liver 'omics gene lists versus only $18 \%$ of text-mining gene lists.

- From the text-mining-based gene lists, CTD and MalaCards strongly outperform GATACA and LoMA gene lists as demonstrated by a difference in the average number of significant gene lists between these databases of up to sevenfold.

- The difference in performance within the text-mining databases is illustrated by the low overlap between gene lists from different databases but representing the same hepatotoxic condition.

- For biomarker development, gene lists derived from human liver 'omics and the CTD and MalaCards databases all seem promising.

\section{Gene list visualization in pathway format}

- Potentially interesting gene lists can be visualized in pathway format using network creation tools that show the interactions between genes.

\section{Conclusion \& future perspective}

" The approach shown here offers a way to generate pathway-level signature profiles relevant for hepatotoxicity that will be of use to the research community.

- By continuing to expand the number of studies, chemicals and associated profiles one can maintain and perpetuate an extensive and up-to-date repository of (hepato)toxicity.

- Exploiting in a dedicated manner existing and openly accessible databases seems promising in providing a venue for translational research in toxicology and biomarker development. 
viral hepatitis [93]. In addition, pathway modeling of gene lists that are of interest provides insight into the biomolecular background of the exposures, which may also be useful in the clinic. However, to confirm the reliability of biomarkers found using this approach, further validation in other hepatotoxicity-specific data sets is first required and needs to be followed by extensive clinical research. This can prove difficult given the relatively low incidence of idiosyncratic hepatotoxicity, but once developed could screen a multitude of hepatotoxic conditions using a wide-range 'omics-based diagnostic test. This would be an especially relevant tool when considering that some drugs can cause different patterns of hepatic injury between individuals [39]. Since idiosyncratic hepatotoxicity has and will continue to be the subject of extensive investigation using 'omics-based approaches, the next decade will undoubtedly provide crucial insight to minimize its occurrence and overcome the lack of standardized criteria or specific gold-standard diagnostic tests. By continuing to expand the number of studies, chemicals and associated profiles one can maintain and perpetuate an extensive and up-to-date repository of (hepato) toxicity with applications in premarket DILI prediction, biomolecular understanding and clinical diagnosis.

In summary, the approach presented in this review, exploiting in a dedicated manner existing and openly accessible databases, seems promising in providing a venue for translational research in toxicology and biomarker development.

\section{Acknowledgements}

The authors would like to thank A Dutta from the Maastricht University BiGCaT department for her contributions to the data analysis.

\section{Financial \& competing interests disclosure}

This work was supported by diXa, a part of the EU Seventh Framework Programme, under grant agreement number RI-283775. The authors have no other relevant affiliations or financial involvement with any organization or entity with a financial interest in or financial conflict with the subject matter or materials discussed in the manuscript apart from those disclosed.

No writing assistance was utilized in the production of this manuscript.

\section{References}

Papers of special note have been highlighted as:

- of interest

" " of considerable interest

1 Shapiro MA, Lewis JH. Causality assessment of drug-induced hepatotoxicity: promises and pitfalls. Clin. Liver Dis. 11(3), 477-505 (2007).

- 2 Stirnimann G, Kessebohm K, Lauterburg B. Liver injury caused by drugs: an update. Swiss Med. Wkly 140, w13080 (2010).

- Review paper on idiosyncratic drug-induced liver injury, its causes and its unpredictability.

-3 Tarantino G, Di Minno MN, Capone D. Drug-induced liver injury: is it somehow foreseeable? World J. Gastroerentol. 15(23), 2817-2833 (2009).

4 Bjornsson E. Drug-induced liver injury: Hy's rule revisited. Clin. Pharmacol. Ther. 79(6), 521-528 (2006).

-5 Chang CY, Schiano TD. Review article: drug hepatotoxicity. Aliment. Pharmacol. Ther. 25(10), 1135-1151 (2007).

-6 Chitturi S, George J. Hepatotoxicity of commonly used drugs: nonsteroidal antiinflammatory drugs, antihypertensives, antidiabetic agents, anticonvulsants, lipidlowering agents, psychotropic drugs. Semin. Liver Dis. 22(2), 169-183 (2002).
Galan MV, Potts JA, Silverman AL, Gordon SC. The burden of acute nonfulminant druginduced hepatitis in a United States tertiary referral center [corrected]. J. Clin.

Gastroenterol. 39(1), 64-67 (2005).

8 Russo MW, Galanko JA, Shrestha R, Fried MW, Watkins P. Liver transplantation for acute liver failure from drug induced liver injury in the United States. Liver Transpl. 10(8), 1018-1023 (2004).

\9 Sgro C, Clinard F, Ouazir K et al. Incidence of drug-induced hepatic injuries: a French population-based study. Hepatology 36(2), 451-455 (2002).

10 Larson AM, Polson J, Fontana RJ et al. Acetaminophen-induced acute liver failure: results of a United States multicenter, prospective study. Hepatology 42(6), 1364-1372 (2005).

- 11 Lee WM, Squires RH Jr, Nyberg SL, Doo E, Hoofnagle JH. Acute liver failure: Summary of a workshop. Hepatology 47(4), 1401-1415 (2008).

12 Russmann S, Kullak-Ublick GA, Grattagliano I. Current concepts of mechanisms in drug-induced hepatotoxicity. Curr. Med. Chem. 16(23), 3041-3053 (2009).

$\checkmark 13$ Hinson JA, Roberts DW, James LP. Mechanisms of acetaminophen-induced liver necrosis. Handb. Exp. Pharmacol. (196), 369-405 (2010).

14 Bell LN, Chalasani N. Epidemiology of idiosyncratic drug-induced liver injury. Semin. Liver Dis. 29(4), 337-347 (2009).

- 15 Bissell DM, Gores GJ, Laskin DL, Hoofnagle JH. Drug-induced liver injury: mechanisms and test systems. Hepatology 33(4), 1009-1013 (2001).

16 Lee WM. Drug-induced hepatotoxicity. N. Engl. J. Med. 349(5), 474-485 (2003).

17 Dambach DM, Andrews BA, Moulin F. New technologies and screening strategies for hepatotoxicity: use of in vitro models. Toxicol. Pathol. 33(1), 17-26 (2005).

- 18 Ostapowicz G, Fontana RJ, Schiodt FV et al. Results of a prospective study of acute liver failure at 17 tertiary care centers in the United States. Ann. Intern. Med. 137(12), 947-954 (2002).

19 Ghabril M, Chalasani N, Bjornsson E. Druginduced liver injury: a clinical update. Curr. Opin. Gastroenterol. 26(3), 222-226 (2010).

20 Fromenty B. Drug-induced liver injury in obesity. J. Hepatol. 58(4), 824-826 (2013).

-21 Lasser KE, Allen PD, Woolhandler SJ, Himmelstein DU, Wolfe SM, Bor DH. Timing of new black box warnings and withdrawals for prescription medications. JAMA 287(17), 2215-2220 (2002). 
- Provides an overview of the frequency and timing of discovery of new adverse drug reactions and concludes that the safety of new agents cannot be known with certainty until a drug has been on the market for many years.

-22 Andrade RJ, Camargo R, Lucena MI, Gonzalez-Grande R. Causality assessment in drug-induced hepatotoxicity. Expert Opin. Drug Saf. 3(4), 329-344 (2004).

-23 Andrade RJ, Robles M, Fernandez-Castaner A, Lopez-Ortega S, Lopez-Vega MC, Lucena MI. Assessment of drug-induced hepatotoxicity in clinical practice: a challenge for gastroenterologists. World J. Gastroerentol. 13(3), 329-340 (2007).

-24 Egan WJ, Zlokarnik G, Grootenhuis PDJ. In silico prediction of drug safety: despite progress there is abundant room for improvement. Drug Discov. Today Technol. 1(4), 381-387 (2004)

- 25 Greaves P, Williams A, Eve M. First dose of potential new medicines to humans: how animals help. Nat. Rev. Drug Discov. 3(3), 226-236 (2004)

-26 Metushi IG, Nakagawa T, Uetrecht J. Direct oxidation and covalent binding of isoniazid to rodent liver and human hepatic microsomes: humans are more like mice than rats. Chem. Res. Toxicol. 25(11), 2567-2576 (2012).

27 Metushi IG, Uetrecht J. Lack of liver injury in Wistar rats treated with the combination of isoniazid and rifampicin. Mol. Cell. Biochem. doi:10.1007/s11010-013-1864-7 (2013) (Epub ahead of print).

-28 Olson H, Betton G, Robinson D et al. Concordance of the toxicity of pharmaceuticals in humans and in animals. Regul. Toxicol. Pharmacol. 32(1), 56-67 (2000).

-29 Olson H, Betton G, Stritar J, Robinson D. The predictivity of the toxicity of pharmaceuticals in humans from animal data - an interim assessment. Toxicol. Lett. 102-103, 535-538 (1998).

- 30 Shih H, Pickwell GV, Guenette DK, Bilir B, Quattrochi LC. Species differences in hepatocyte induction of CYP1A1 and CYP1A2 by omeprazole. Hum. Exp. Toxicol. 18(2), 95-105 (1999).

- 31 Suter L, Schroeder S, Meyer K et al. EU framework 6 project: predictive toxicology (PredTox) - overview and outcome. Toxicol. Appl. Pharmacol. 252(2), 73-84 (2011).

-11-Overview and outcome report of the PredTox program indicating that 'omics technologies provide additional information that can help toxicologists to make better informed decisions during exploratory toxicological studies.
\$2 Uetrecht J. Role of animal models in the study of drug-induced hypersensitivity reactions. AAPS J. 7(4), e914-e921 (2005).

33 Van Summeren A, Renes J, van Delft JH, Kleinjans JC, Mariman EC. Proteomics in the search for mechanisms and biomarkers of drug-induced hepatotoxicity. Toxicol. In Vitro 26(3), 373-385 (2012).

- 34 Au JS, Navarro VJ, Rossi S. Review article: drug-induced liver injury - its pathophysiology and evolving diagnostic tools. Aliment. Pharmacol. Ther. 34(1), 11-20 (2011).

35 Casciano DA. The use of genomics in model in vitro systems. Adv. Exp. Med. Biol. 745 , 210-220 (2012).

36 Przybylak KR, Cronin MT. In silico models for drug-induced liver injury - current status. Expert Opin. Drug Metab. Toxicol. 8(2), 201-217 (2012).

"- Explores the current status of existing in silico models predicting hepatotoxicity.

37 van Meer S, de Man RA, Siersema PD, van Erpecum KJ. Surveillance for hepatocellular carcinoma in chronic liver disease: evidence and controversies. World J. Gastroerentol. 19(40), 6744-6756 (2013).

38 Lazaridis KN, Gores GJ. Primary sclerosing cholangitis and cholangiocarcinoma. Semin. Liver Dis. 26(1), 42-51 (2006).

- 39 Aithal GP, Watkins PB, Andrade RJ et al. Case definition and phenotype standardization in drug-induced liver injury. Clin. Pharmacol. Ther. 89(6), 806-815 (2011).

40 Gao B, Bataller R. Alcoholic liver disease: pathogenesis and new therapeutic targets. Gastroenterology 141(5), 1572-1585 (2011).

41 Davis AP, Murphy CG, Johnson R et al. The Comparative Toxicogenomics Database: update 2013. Nucleic Acids Res. 41(Database issue), D1104-D1114 (2013).

42 Mattingly CJ, Colby GT, Forrest JN, Boyer JL. The Comparative Toxicogenomics Database (CTD). Environ. Health Perspect. 111(6), 793-795 (2003).

43 Buchkremer S, Hendel J, Krupp M et al. Library of molecular associations: curating the complex molecular basis of liver diseases. $B M C$ Genomics 11, 189 (2010).

44 Rappaport N, Nativ N, Stelzer G et al. MalaCards: an integrated compendium for diseases and their annotation. Database (Oxford) 2013, bat018 (2013).

-45 Stelzer G, Dalah I, Stein TI et al. In-silico human genomics with GeneCards. Hum. Genomics 5(6), 709-717 (2011).

46 Barrett T, Edgar R. Gene expression omnibus: microarray data storage, submission, retrieval, and analysis. Methods Enzymol. 411, 352-369 (2006).
Brazma A, Parkinson H, Sarkans U et al. ArrayExpress - a public repository for microarray gene expression data at the EBI. Nucleic Acids Res. 31(1), 68-71 (2003).

48 Affò S, Dominguez M, Lozano JJ et al. Transcriptome analysis identifies TNF superfamily receptors as potential therapeutic targets in alcoholic hepatitis. Gut 62(3), 452-460 (2013).

49 Andersen JB, Spee B, Blechacz BR et al. Genomic and genetic characterization of cholangiocarcinoma identifies therapeutic targets for tyrosine kinase inhibitors. Gastroenterology 142(4), 1021-1031.e15 (2012).

-50 Bourd-Boittin K, Bonnier D, Leyme A et al. Protease profiling of liver fibrosis reveals the ADAM metallopeptidase with thrombospondin type 1 motif, 1 as a central activator of transforming growth factor beta. Hepatology 54(6), 2173-2184 (2011).

-51 Caillot F, Hiron M, Goria O et al. Novel serum markers of fibrosis progression for the follow-up of hepatitis C virus-infected patients. Am. J. Pathol. 175(1), 46-53 (2009).

52 Lake AD, Novak P, Fisher CD et al. Analysis of global and absorption, distribution, metabolism, and elimination gene expression in the progressive stages of human nonalcoholic fatty liver disease. Drug Metab. Dispos. 39(10), 1954-1960 (2011).

53 Liu W, Baker SS, Baker RD, Nowak NJ, Zhu L. Upregulation of hemoglobin expression by oxidative stress in hepatocytes and its implication in nonalcoholic steatohepatitis. PLoS ONE 6(9), e24363 (2011)

54 Marshall A, Lukk M, Kutter C, Davies S, Alexander G, Odom DT. Global gene expression profiling reveals SPINK1 as a potential hepatocellular carcinoma marker. PLoS ONE 8(3), e59459 (2013).

55 Nissim O, Melis M, Diaz G et al. Liver regeneration signature in hepatitis $\mathrm{B}$ virus (HBV)-associated acute liver failure identified by gene expression profiling. PLoS ONE 7(11), e49611 (2012).

56 Onomoto K, Morimoto S, Kawaguchi T et al. Dysregulation of IFN system can lead to poor response to pegylated interferon and ribavirin therapy in chronic hepatitis C. PLoS ONE 6(5), e19799 (2011)

- 57 Rasmussen AL, Tchitchek N, Susnow NJ et al. Early transcriptional programming links progression to hepatitis $\mathrm{C}$ virus-induced severe liver disease in transplant patients. Hepatology 56(1), 17-27 (2012).

-58 Sia D, Hoshida Y, Villanueva A et al. Integrative molecular analysis of intrahepatic cholangiocarcinoma reveals 2 classes that have different outcomes. Gastroenterology 144(4), 829-840 (2013). 
-59 Starmann J, Falth M, Spindelbock W et al. Gene expression profiling unravels cancerrelated hepatic molecular signatures in steatohepatitis but not in steatosis. PLoS ONE 7(10), e46584 (2012).

60 Staten NR, Welsh EA, Sidik K et al. Multiplex transcriptional analysis of paraffin-embedded liver needle biopsy from patients with liver fibrosis. Fibrogenesis Tissue Repair 5(1), 21 (2012).

-61 Wurmbach E, Chen YB, Khitrov G et al. Genome-wide molecular profiles of HCVinduced dysplasia and hepatocellular carcinoma. Hepatology 45(4), 938-947 (2007).

62 Shannon P, Markiel A, Ozier O et al. Cytoscape: a software environment for integrated models of biomolecular interaction networks. Genome Res. 13(11), 2498-2504 (2003).

63 Smoot ME, Ono K, Ruscheinski J, Wang PL, Ideker T. Cytoscape 2.8: new features for data integration and network visualization. Bioinformatics 27(3), 431-432 (2011).

64 Salomonis N, Hanspers K, Zambon AC et al. GenMAPP 2: new features and resources for pathway analysis. BMC Bioinformatics 8, 217 (2007).

65 van Iersel MP, Kelder T, Pico AR et al. Presenting and exploring biological pathways with PathVisio. BMC Bioinformatics 9, 399 (2008).

-66 Uehara T, Ono A, Maruyama T et al. The Japanese toxicogenomics project: application of toxicogenomics. Mol. Nutr. Food Res. 54(2), 218-227 (2010).

- Explores potential applications and features of the Japanese Toxicogenomics Project, which includes the TG-GATEs database.

67 Ma MK, Woo MH, Mcleod HL. Genetic basis of drug metabolism. Am. J. Health Syst. Pharm. 59(21), 2061-2069 (2002).

68 Zhou SF, Liu JP, Chowbay B. Polymorphism of human cytochrome $\mathrm{P} 450$ enzymes and its clinical impact. Drug Metab. Rev. 41(2), 89-295 (2009).

-69 Chen M, Vijay V, Shi Q, Liu Z, Fang H, Tong W. FDA-approved drug labeling for the study of drug-induced liver injury. Drug Discov. Today 16(15-16), 697-703 (2011).

-70 Brandon EF, Raap CD, Meijerman I, Beijnen $\mathrm{JH}$, Schellens JH. An update on in vitro test methods in human hepatic drug biotransformation research: pros and cons. Toxicol. Appl. Pharmacol. 189(3), 233-246 (2003).

71 Gomez-Lechon MJ, Castell JV, Donato MT. An update on metabolism studies using human hepatocytes in primary culture. Expert Opin. Drug Metab. Toxicol. 4(7), 837-854 (2008).
72 Gomez-Lechon MJ, Donato MT, Castell JV, Jover R. Human hepatocytes in primary culture: the choice to investigate drug metabolism in man. Curr. Drug Metab. 5(5), 443-462 (2004).

73 Guillouzo A. Liver cell models in in vitro toxicology. Environ. Health Perspect. 106(Suppl. 2), 511-532 (1998).

74 Olsavsky KM, Page JL, Johnson MC, Zarbl H, Strom SC, Omiecinski CJ. Gene expression profiling and differentiation assessment in primary human hepatocyte cultures, established hepatoma cell lines, and human liver tissues. Toxicol. Appl. Pharmacol. 222(1), 42-56 (2007).

75 Guo L, Dial S, Shi L et al. Similarities and differences in the expression of drugmetabolizing enzymes between human hepatic cell lines and primary human hepatocytes. Drug Metab. Dispos. 39(3), 528-538 (2011).

=- Assessment of gene expression spectra of drug-metabolizing enzymes and transporters, showing that primary human hepatocytes resemble human liver more closely than several hepatic cell lines.

76 Hart SN, Li Y, Nakamoto K, Subileau EA, Steen D, Zhong XB. A comparison of whole genome gene expression profiles of HepaRG cells and HepG2 cells to primary human hepatocytes and human liver tissues. Drug Metab. Dispos. 38(6), 988-994 (2010).

77 Jetten MJ, Kleinjans JC, Claessen SM, Chesne C, van Delft JH. Baseline and genotoxic compound induced gene expression profiles in HepG2 and HepaRG compared with primary human hepatocytes. Toxicol. In Vitro 27(7), 2031-2040 (2013).

78 Parkinson A, Kazmi F, Buckley DB, Yerino P, Ogilvie BW, Paris BL. System-dependent outcomes during the evaluation of drug candidates as inhibitors of cytochrome P450 (CYP) and uridine diphosphate glucuronosyltransferase (UGT) enzymes: human hepatocytes versus liver microsomes versus recombinant enzymes. Drug Metab. Pharmacokinet. 25(1), 16-27 (2010).

79 Wilkening S, Stahl F, Bader A. Comparison of primary human hepatocytes and hepatoma cell line Hepg2 with regard to their biotransformation properties. Drug Metab. Dispos. 31(8), 1035-1042 (2003).

80 Gerets HH, Tilmant K, Gerin B et al. Characterization of primary human hepatocytes, HepG2 cells, and HepaRG cells at the mRNA level and CYP activity in response to inducers and their predictivity for the detection of human hepatotoxins. Cell Biol. Toxicol. 28(2), 69-87 (2012).

- Investigation of the predictivity of HepG2, HepaRG and primary human hepatocytes to detect hepatotoxins, showing that none of these in vitro models yield desirable sensitivities.

81 Li AP. Human hepatocytes: isolation, cryopreservation and applications in drug development. Chem. Biol. Interact. 168(1), 16-29 (2007).

-82 Gao J, Ade AS, Tarcea VG et al. Integrating and annotating the interactome using the MiMI plugin for cytoscape. Bioinformatics 25(1), 137-138 (2009).

83 Jayapandian M, Chapman A, Tarcea VG et al. Michigan Molecular Interactions (MiMI): putting the jigsaw puzzle together. Nucleic Acids Res. 35(Database issue), D566-D571 (2007).

84 Tarcea VG, Weymouth T, Ade A et al. Michigan molecular interactions $\mathrm{r} 2$ : from interacting proteins to pathways. Nucleic Acids Res. 37(Database issue), D642-D646 (2009).

85 Kelder T, van Iersel MP, Hanspers K et al. WikiPathways: building research communities on biological pathways. Nucleic Acids Res. 40(Database issue), D1301-D1307 (2012).

-86 Abdel-Bakky MS, Hammad MA, Walkerit LA, Ashfaqi MK. Developing and characterizing a mouse model of hepatotoxicity using oral pyrrolizidine alkaloid (monocrotaline) administration, with potentiation of the liver injury by coadministration of LPS. Nat. Prod. Commun. 5(9), 1457-1462 (2010).

87 De Bruyn T, Chatterjee S, Fattah S et al. Sandwich-cultured hepatocytes: utility for in vitro exploration of hepatobiliary drug disposition and drug-induced hepatotoxicity. Expert Opin. Drug Metab. Toxicol. 9(5), 589-616 (2013).

88 Doktorova TY, Yildirimman R, Vinken M et al. Transcriptomic responses generated by hepatocarcinogens in a battery of liver-based in vitro models. Carcinogenesis 34(6), 1393-1402 (2013).

89 Hill A, Mesens N, Steemans M, Xu JJ, Aleo MD. Comparisons between in vitro whole cell imaging and in vivo zebrafish-based approaches for identifying potential human hepatotoxicants earlier in pharmaceutical development. Drug Metab. Rev. 44(1), 127-140 (2012).

90 Kostadinova R, Boess F, Applegate D et al. A long-term three dimensional liver co-culture system for improved prediction of clinically relevant drug-induced hepatotoxicity. Toxicol. Appl. Pharmacol. 268(1), 1-16 (2013).

-91 Lake BG, Price RJ. Evaluation of the metabolism and hepatotoxicity of xenobiotics utilizing precision-cut slices. Xenobiotica 43(1), 41-53 (2013). 
92 Pery AR, Brochot C, Zeman FA et al. Prediction of dose-hepatotoxic response in humans based on toxicokinetic/toxicodynamic modeling with or without in vivo data: a case study with acetaminophen. Toxicol. Lett. 220(1), 26-34 (2013).

93 Hussaini SH, Farrington EA. Idiosyncratic drug-induced liver injury: an update on the 2007 overview. Expert Opin. Drug Saf. 13(1), 67-81 (2013).

\section{Websites}

101 Larson AM. Drugs and the liver: patterns of drug-induced liver injury. UpToDate (2013). www.uptodate.com/contents/drugs-and-theliver-patterns-of-drug-induced-liver-injury
102 Mehta N, Ozick LA, Gbadehan E. Druginduced hepatotoxicity. Medscape (2012). http://emedicine.medscape.com/ article/169814-overview

103 diXa.

www.dixa-fp7.eu (Accessed 10 October 2013)

104 The Comparative Toxicogenomics Database (CTD). http://ctdbase.org (Accessed 10 July 2013)

105 GATACA.

https://gataca.cchmc.org/gataca (Accessed 15 July 2013)

106 Medicalgenomics. LoMA. http://medicalgenomics.org/loma (Accessed 11 July 2013)
107 MalaCards - human disease database. www.malacards.org (Accessed 17 July 2013)

108 GeneCards $^{\circledR}$ - GeneALaCart Beta. www.genecards.org/BatchQueries/index. php

(Accessed 19 August 2013)

109 EMBl-EBI. ArrayExpress. www.ebi.ac.uk/arrayexpress (Accessed 19 September 2013)

110 NCBI. Gene Expression Omnibus. www.ncbi.nlm.nih.gov/geo (Accessed 19 September 2013)

111 NIBIO. Open TG-GATEs. http://toxico.nibio.go.jp (Accessed 23 August 2013) 